\title{
Gaze holding deficits discriminate early from late onset cerebellar degeneration
}

\author{
Alexander A. Tarnutzer ${ }^{1}$ (I) $\cdot$ K. P. Weber ${ }^{1,2} \cdot$ B. Schuknecht ${ }^{3} \cdot$ D. Straumann ${ }^{1}$. \\ S. Marti $^{1}$ - G. Bertolini ${ }^{1}$
}

Received: 26 March 2015/Revised: 29 April 2015/Accepted: 29 April 2015/Published online: 16 May 2015

(C) Springer-Verlag Berlin Heidelberg 2015

\begin{abstract}
The vestibulo-cerebellum calibrates the output of the inherently leaky brainstem neural velocity-to-position integrator to provide stable gaze holding. In healthy humans small-amplitude centrifugal nystagmus is present at extreme gaze-angles, with a non-linear relationship between eye-drift velocity and eye eccentricity. In cerebellar degeneration this calibration is impaired, resulting in pathological gaze-evoked nystagmus (GEN). For cerebellar dysfunction, increased eye drift may be present at any gaze angle (reflecting pure scaling of eye drift found in controls) or restricted to far-lateral gaze (reflecting changes in shape of the non-linear relationship) and resulting eyed-drift patterns could be related to specific disorders. We recorded horizontal eye positions in 21 patients with cerebellar neurodegeneration (gaze-angle $= \pm 40^{\circ}$ ) and clinically confirmed GEN. Eye-drift velocity, linearity and symmetry of drift were determined. MR-images were assessed for cerebellar atrophy. In our patients, the relation between eye-drift velocity and gaze eccentricity was non-linear, yielding (compared to controls) significant GEN at gazeeccentricities $\geq 20^{\circ}$. Pure scaling was most frequently observed $(n=10 / 18)$, followed by pure shape-changing $(n=4 / 18)$ and a mixed pattern $(n=4 / 18)$. Pure shapechanging patients were significantly $(p=0.001)$ younger
\end{abstract}

Alexander A. Tarnutzer

alalexander.tarnutzer@access.uzh.ch

1 Department of Neurology, University Hospital Zurich, University of Zurich, Frauenklinikstr. 26, 8091 Zurich, Switzerland

2 Department of Ophthalmology, University Hospital Zurich, University of Zurich, Zurich, Switzerland

3 Medizinisch Radiologisches Institut, Zurich, Switzerland at disease-onset compared to pure scaling patients. Atrophy centered around the superior/dorsal vermis, flocculus/paraflocculus and dentate nucleus and did not correlate with the specific drift behaviors observed. Eye drift in cerebellar degeneration varies in magnitude; however, it retains its non-linear properties. With different drift patterns being linked to age at disease-onset, we propose that the gaze-holding pattern (scaling vs. shape-changing) may discriminate early- from late-onset cerebellar degeneration. Whether this allows a distinction among specific cerebellar disorders remains to be determined.

Keywords Cerebellar atrophy $\cdot$ Magnetic resonance . Neurodegenerative disorders - Ocular motor control . Velocity-to-position integrator

\section{Introduction}

Gaze-evoked nystagmus (GEN) is defined as a sustained centrifugal nystagmus [1] reflecting the brain's response when eccentric gaze cannot be maintained and as a result the eyes drift back towards straight-ahead position [2, 3]. Horizontal gaze holding is provided by a brainstem horizontal neural velocity-to-position integrator $[4,5]$ located in the nucleus prepositus hypoglossi and the medial vestibular nucleus $[3,6]$. Robinson proposed that the neural velocity-to-position integrator is inherently "leaky" and as a result requires cerebellar input to calibrate its output precisely in proportion to eye position [5]. Impaired gaze holding secondary to such "leaky" integration has been demonstrated in humans with acute [7, 8] and chronic [912] cerebellar disease and in primates after flocculectomy [13] or (hemi-)cerebellectomy [14-16]. Centripetal eyedrift, therefore, is considered an essential clinical sign of a 
deficient brainstem neural velocity-to-position integrator or its cerebellar modulatory structures.

At extreme angles of eccentric gaze, most healthy human subjects show a small-amplitude horizontal centripetal eye drift, termed end-point nystagmus (EPN) [17, 18]. Gaze shifts to moderate horizontal eccentricities evoke only very weak centripetal eye drift in healthy subjects, as cerebellar control sufficiently compensates for the inherent leakiness of the brainstem gaze-holding network $[4,5,13$, 15]. We recently assessed horizontal gaze holding (range $= \pm 40^{\circ}$ ) and quantified EPN in healthy human subjects, demonstrating over-proportional increases in eye drift for gaze angles $>20^{\circ}$ [19]. We hypothesized that the gaze holding system is optimized to behave linearly within a given range, but loses this behavior at larger gaze angles, resulting in a non-linear behavior even in healthy human subjects.

In view of the non-linear relationship between eye-drift velocity and horizontal eye position and the physiological leakiness of gaze holding at extreme gaze-angles, we predict profound non-linear eye drift at eccentric gaze in patients with insufficient cerebellar control of the brainstem neural integrator. Different mechanisms (see also Fig. 1) may explain increased eye drift: it may reflect (1) a pure scaling effect of eye drift, i.e. drift velocity increases by a given factor independently from the gaze angle, (2) that gaze becomes deficient only beyond a certain gaze-angle, reflecting a change in shape of the position-velocity curve rather than pure scaling, (3) a combination of mechanisms one and two. Possibly these mechanisms reflect a continuum of conditions, with scaling representing more advanced cerebellar disease with gaze-holding being impaired at small gaze-angles also or are "diseasespecific".

\section{Materials and methods}

\section{Subjects}

Twenty-one patients $[63.0 \pm 13.3$ years, average \pm 1 standard deviation (SD)] with various adult-onset neurodegenerative cerebellar disorders participated (Table 1). All of them displayed typical ocular motor signs of cerebellar disease including GEN, which was a prerequisite for inclusion. Written informed consent of all participants was obtained after a full explanation of the experimental procedure. The protocol was approved by the local ethics committee and was in accordance with the ethical standards laid down in the 2013 Declaration of Helsinki for research involving human subjects.

For comparison, gaze-holding data from 20 healthy human subjects $(41 \pm 11$ year old) previously described

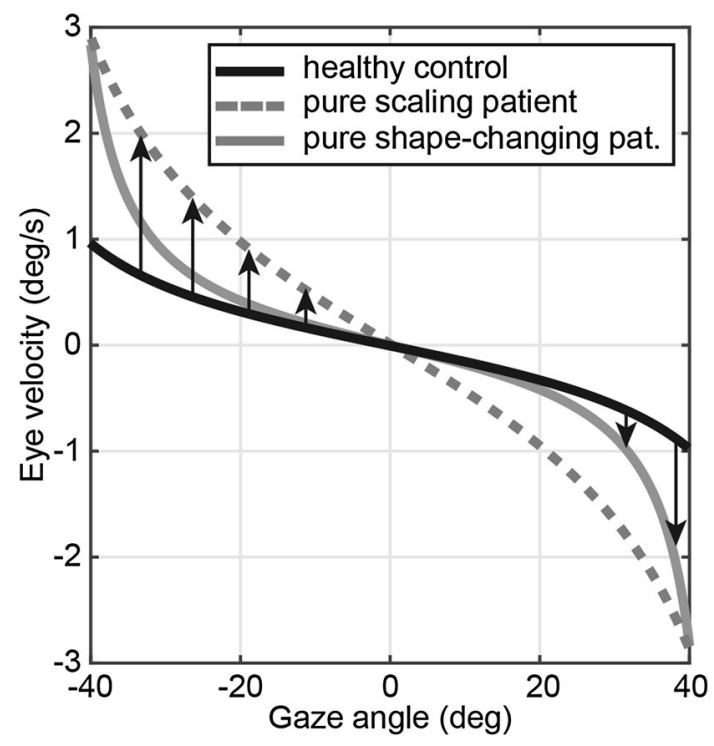

Fig. 1 Illustration of simulated data showing two possible predicted eye drift behaviors in relation to horizontal eye position in patients with neurodegenerative cerebellar disease (gray lines) and comparison with a healthy control subject (black solid line). Increases in eye drift velocity in the patients are indicated by black arrows. In the first patient (gray dashed line) eye velocity values over the entire range of gaze angles are multiplied by a given value, reflecting "pure scaling" behavior. In the second patient (gray solid line), eye drift velocity values remain within normal up to approximately $20^{\circ}-25^{\circ}$ of gaze eccentricity and only for more extreme gaze angles, significant increases in eye drift velocity can be observed, reflecting a "pure shape-changing" behavior of eye drift. In addition, patients may show patterns between pure shape-changing and pure scaling, referred to as scaling and shape-changing behavior

by [19] were used. This control group was intended to represent optimal gaze holding properties and was, therefore, not age-matched.

A neuro-otological examination was performed in all participants. MRI-images previously obtained for clinical purposes were reviewed and the degree of cerebellar atrophy was rated by an experienced neuro-radiologist (BS) that was unaware of the clinical presentation of the patients.

\section{Experimental setting}

All recordings were obtained on a chair mounted on a two (Tönnies D561, Freiburg, Germany) or three (prototype, Acutronic, Jona, Switzerland) servo-controlled motor-driven axes turntable system. Visual stimuli were generated by LEDs at eye level. The head was stabilized in upright position using a head-and-chin rest (Tönnies) or a thermoplastic mask (Acutronic). Horizontal eye movements were recorded in darkness at $220 \mathrm{~Hz}$ with a head-mounted video-oculography device ("EyeSeeCam") [20, 21]. During recordings, one eye was covered with a lens filter, 


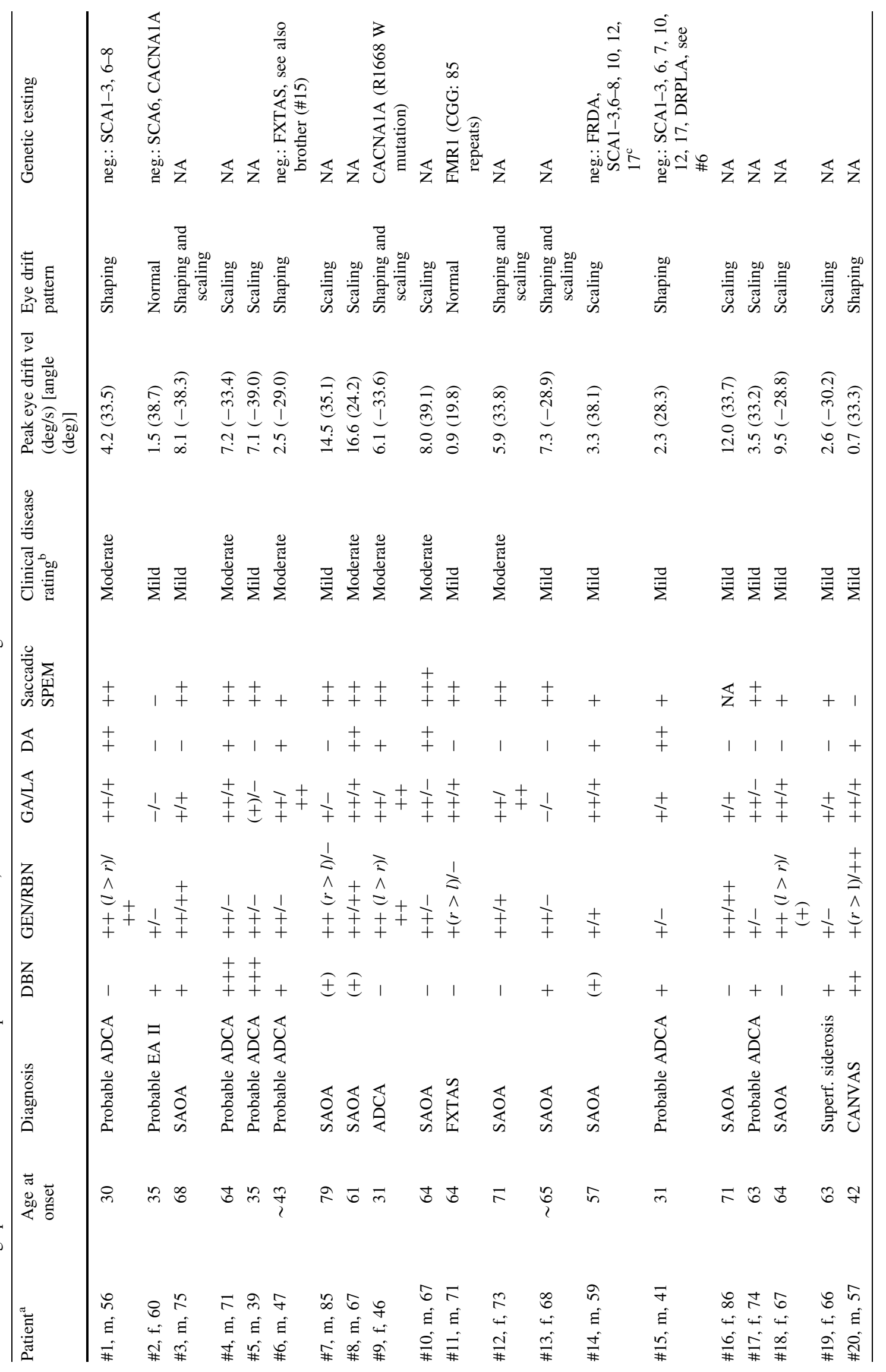




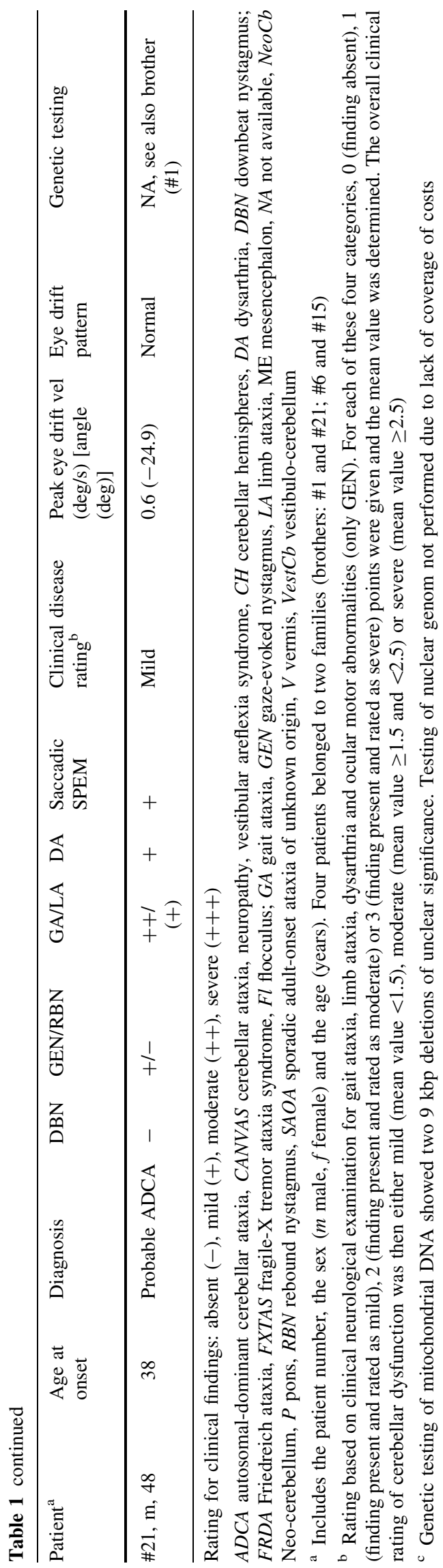

preventing vision but allowing video recording of the eye by the infrared camera.

\section{Experimental procedure}

Participants were asked to look at a briefly flashing $(50 \mathrm{~ms}$ every 2 s) LED without moving the head. The LED was positioned in the range of horizontal gaze eccentricity from $-40^{\circ}$ to $+40^{\circ}$. Each subject was tested in two subsequent runs, changing the order of presentation of the requested gaze eccentricities and for the right and left eye viewing separately. The LED always started straight-ahead and slowly displaced $\left(0.5 \%\right.$ s, i.e. by $1^{\circ}$ at every flash) in one direction (random order) up to the maximum eccentricity before changing direction and completing the full cycle (up to $40^{\circ}$ of eccentricity into the direction of the viewing eye and up to $20^{\circ}$ of eccentricity into the opposite direction as the target gets covered by the nose at higher eccentricities). Both eyes were recorded simultaneously. A similar "slowmoving dot" paradigm had been used for data acquisition of 20 healthy human subjects [19], with the only difference that no eye was covered and that the flashing LED covered the whole range of $80^{\circ}$ tested $\left( \pm 40^{\circ}\right)$ in each trial. Compared to the classic approach of quantifying gaze-holding deficits by use of saccades to certain, selected angles of gaze-eccentricity, the slow-moving dot paradigm has the advantage of much higher spatial resolution $\left(1^{\circ}\right.$ of horizontal gaze eccentricity), potentially allowing the identification of more subtle changes in gaze-holding deficits and superior fitting of mathematical models. In order to assess the impact of the selected paradigm on measured gaze-holding deficits, we also obtained the classic "saccade-based" paradigm in 13/21 patients (\#3-6, 12-15, 17-21). In this paradigm desired gaze position was reached by a saccade from straight-ahead position. GEN was recorded at selected angles of eccentricity $\left(-30^{\circ}\right.$ to $+30^{\circ}$, steps $=5^{\circ}$, random order) indicated by a stationary flashing red dot (50 ms every $2 \mathrm{~s}$ for $6 \mathrm{~s})$.

\section{Data analysis}

Data analysis was done using interactive programs written in MATLAB (The Mathworks, Natick, MA). Velocity traces were obtained as the derivative of horizontal eye position traces. Saccades and blinks were removed interactively (see [19] for a detailed description). The resulting data points were assigned to one of 17 non-overlapping bins (bin-width $=5^{\circ}$ ), covering together $\pm 40^{\circ}$ of gaze eccentricity. Additionally, each participant's instantaneous eye velocity was smoothed as a function of eye eccentricity using weighted linear least-squares robust regression (smooth.m, "rloess" algorithm, MATLAB) and interpolated at every $0.1^{\circ}$ [19]. Values from all dependent 
variables were evaluated for normality using Lillierfors test. Whenever normality was not confirmed, median and median absolute deviations (MAD) were used as descriptive statistic parameters instead of the mean and SD. Bonferroni correction was applied when multiple comparisons were made.

\section{Mathematical modeling}

The instantaneous eye velocity of each subject from both groups was independently fitted with the following functions:

$V=m \times E+c_{1}$

$V=\frac{k_{2}}{k_{1}} \tan \left(k_{1} \times E\right)+c_{2}$

In both Eqs. (1) and (2), $V$ is the instantaneous eye velocity recorded while $E$ is the corresponding instantaneous eye position. The linear slope $m$, the coefficients $k_{1}$ and $k_{2}$ and the offsets $c_{1}$ and $c_{2}$ were optimized with a least-square algorithm. Equation (1) specifies a linear dependency of the eye drift velocity on eye position. Equation (2) is a modified version of the tangent function proposed previously [19]. Changes in $k_{1}$ only cause changes in the shape of the tangent function, while changes in $k_{2}$ lead to scaling up the velocity, preserving the shape.

\section{Results}

\section{Clinical and brain MRI findings}

Details of the clinical neurological examination can be found in Table 1. Ocular motor abnormalities (besides GEN) included downbeat-nystagmus $(n=13)$, reboundnystagmus $(n=9)$ and saccadic smooth-pursuit $(n=18)$. MRI demonstrated cerebellar atrophy in 19/20 patients (see Table 2 for details). Within the vermis, lobules IV-VI $(n=17 / 20)$, VIIa $(n=14 / 20)$ and VIIb $(n=10 / 20)$ were affected most frequently (see Fig. 2a, b for an example), while lobules VIII-X were usually spared. The flocculus/paraflocculus was atrophic in 10/20 patients (Fig. 2c, d). Degenerative changes within the dentate nucleus were observed in 10/20 patients (Fig. 2e, f). Atrophy within the cerebellar hemispheres was most often found in the anterior parts $(n=13 / 20)$.

\section{Gaze holding: peak values, asymmetry and drift patterns}

In Fig. 3 raw eye-position and eye-velocity data are shown for a healthy subject and a patient, illustrating differences in GEN. To emphasize the gaze-dependent eye drift, we plotted eye-drift velocity as a function of gaze eccentricity ['position-velocity (PV) plots']. Three patients with different eye-drift behavior are shown in Fig. 4a-c. In Fig. 4d the gaze-holding properties in patients and controls are compared. For the following analysis, raw instantaneous velocity values were used.

Eye-drift velocity values were assigned to $5^{\circ}$-bins and median drift velocity of each subject in each bin was calculated. Eye-drift velocity was significantly higher in patients for bins centered at $\geq 20^{\circ}(p<0.001$, nonparametric Mann-Whitney-Wilcoxon test) (Table 3).

The median asymmetry ratio of the eye-drift velocities within each pair of corresponding bins from left and right gaze was $1.41(0.23 ; 1 \mathrm{MAD})$, not being significantly ( $p>0.05$, nonparametric Mann-Whitney-Wilcoxon test) different from the healthy controls' median [1.56 (0.34)]. The median of the absolute differences between the corresponding bins in the patient group ranged between $0.66 \%$ $\mathrm{s}(0.31 \% \mathrm{~s})$ at $40^{\circ}$ and $0.11 \% \mathrm{~s}\left(0.08^{\circ} / \mathrm{s}\right)$ at $5^{\circ}$.

The two parameters of the tangent function allowed differentiating between distinct changes of the drift behavior. While the first parameter $\left(k_{1}\right)$ characterized the increase of eye drift at large gaze angles compared to small ones (i.e. refers to its shaping), the second parameter $\left(k_{2}\right)$ described the global amount of eye-drift velocity over the range of gaze angles studied (i.e. refers to its scaling). For the patients, the median $r^{2}$ of the tangent function [0.80 $(0.10)]$ was significantly $(p=0.007)$ higher than the value obtained with a linear function $[0.75(0.14)]$, matching what previously was shown in healthy subjects [19]. We split the $k_{1}-k_{2}$ parameter-space in four subspaces, using the median +1 MAD of $k_{1}$ and $k_{2}$ estimated from the controls as thresholds (Fig. 5a). Four patients showed a significant increase restricted to $k_{1}$; in 10 patients only $k_{2}$ was significantly larger. Four patients exceed the threshold for both parameters.

\section{Correlation between clinical parameters and drift patterns observed}

With age at symptom onset ranging between 30 and 79 years, two clusters could be identified: an early-onset group $(n=8 ; \quad$ range $30-43$ years; average $\pm 1 \mathrm{SD}$ $35.6 \pm 5.0$ years $)$ and a late-onset group $(n=13$; range 57-79 years; average \pm 1 SD $65.5 \pm 5.5$ years). Amongst those patients with early disease-onset, disease duration varied considerably (13.6 \pm 8.4 years; range 4-26 years). We, however, did not observe different drift patterns for early-onset patients as a function of disease duration. Overall, six out of those eight patients showed significant GEN, with the drift pattern being shape-changing in four. Pooling the two clusters, disease duration was significantly 


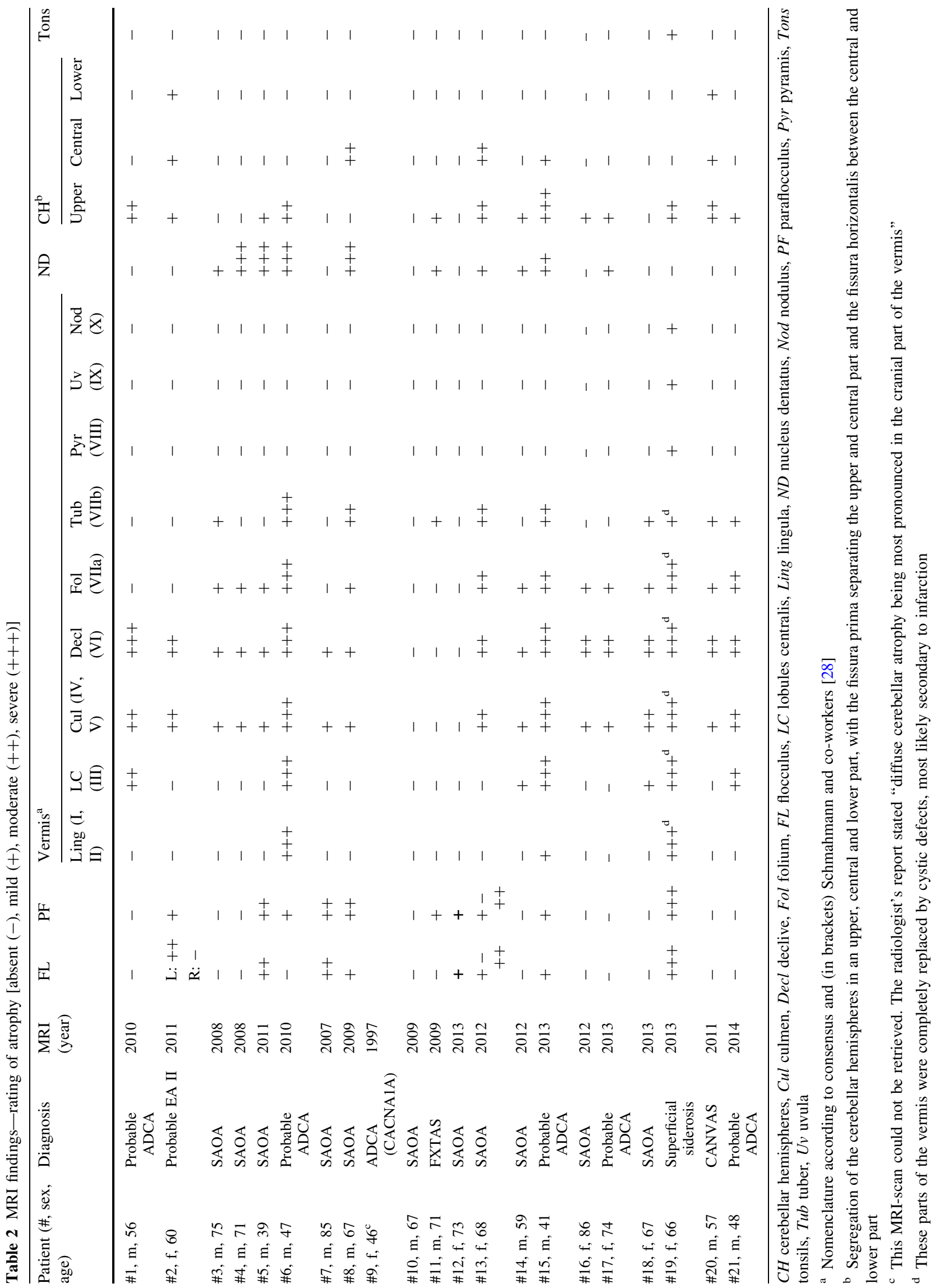



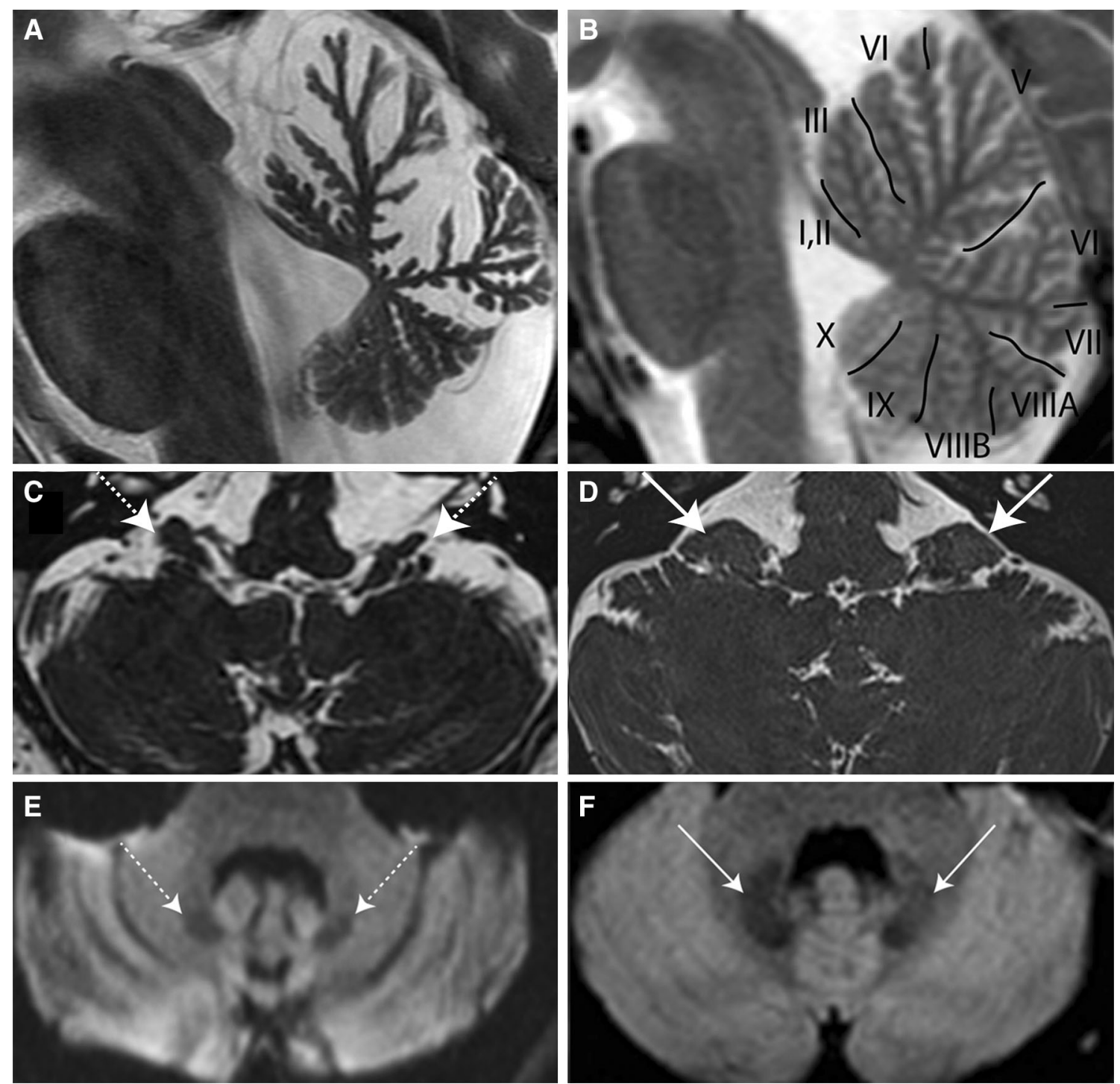

Fig. 2 Atrophic changes in different cerebellar areas-exemplary illustration in single patients (left column) and comparison with normal findings (right column). a Severe atrophy of the superior and dorsal cerebellar vermis with relative sparing of the caudal vermis (patient \#15, sagittal T2-weighted MR-sequence). b Sagittal T2weighted MR-image of the cerebellar vermis in a healthy human control subject with segregation of the different vermal lobuli [28]. c Axial FIESTA (fast imaging employing steady state acquisition) image demonstrating moderate atrophy of the flocculus (indicated by

longer in the pure shape-changing group compared to the pure scaling group $(13.8 \pm 9.3$ vs. $6.0 \pm 4.1$ years; $p=0.046$, two-sample $t$ test). Mean age both at recording $(50.3 \pm 7.6$ vs. $68.1 \pm 13.3$ years; $p=0.028)$ and at symptom onset $(36.5 \pm 7.0 \quad$ vs. $62.1 \pm 11.2$ years; $p=0.001)$ in the pure shape-changing group was significantly lower than that in the pure scaling group.

Other clinical parameters such as frequency of downbeat nystagmus (ranging between 50 and $75 \%$ in patients with GEN) and dysarthria (ranging between 25 and $100 \%$ ) the white dashed arrows) in patient \#12. d Axial FIESTA image in a subject with normal volume of the flocculus (solid white arrow) for comparison. e Axial diffusion-weighted (DWI) imaging showing reduced volume and loss of hypointensity of the dentate nucleus (dashed white arrows) in patient \#6. f Axial DWI illustrating normal volume and signal intensity of the dentate nucleus (solid white arrows) in a healthy human control. Courtesy of MR-images: Department of Neuroradiology, University Hospital Zurich, Switzerland (a, c, f) and MRI Institute, Zurich, Switzerland (b, d, e)

showed either only a trend towards significant differences ( $p=0.093$ for rates of dysarthria in pure shape-changing vs. pure scaling patients, Fisher's exact test) or were not significantly different $(p>0.05)$.

Possibly, a global increase of eye-drift velocity (i.e. pure scaling) as noted in our scaling patients could also be related to a physiological decrease in gaze-holding control with increasing age. However, a correlation analysis (regress.m, built-in Matlab function) between the amount of eye drift and age in our healthy control subjects did not 

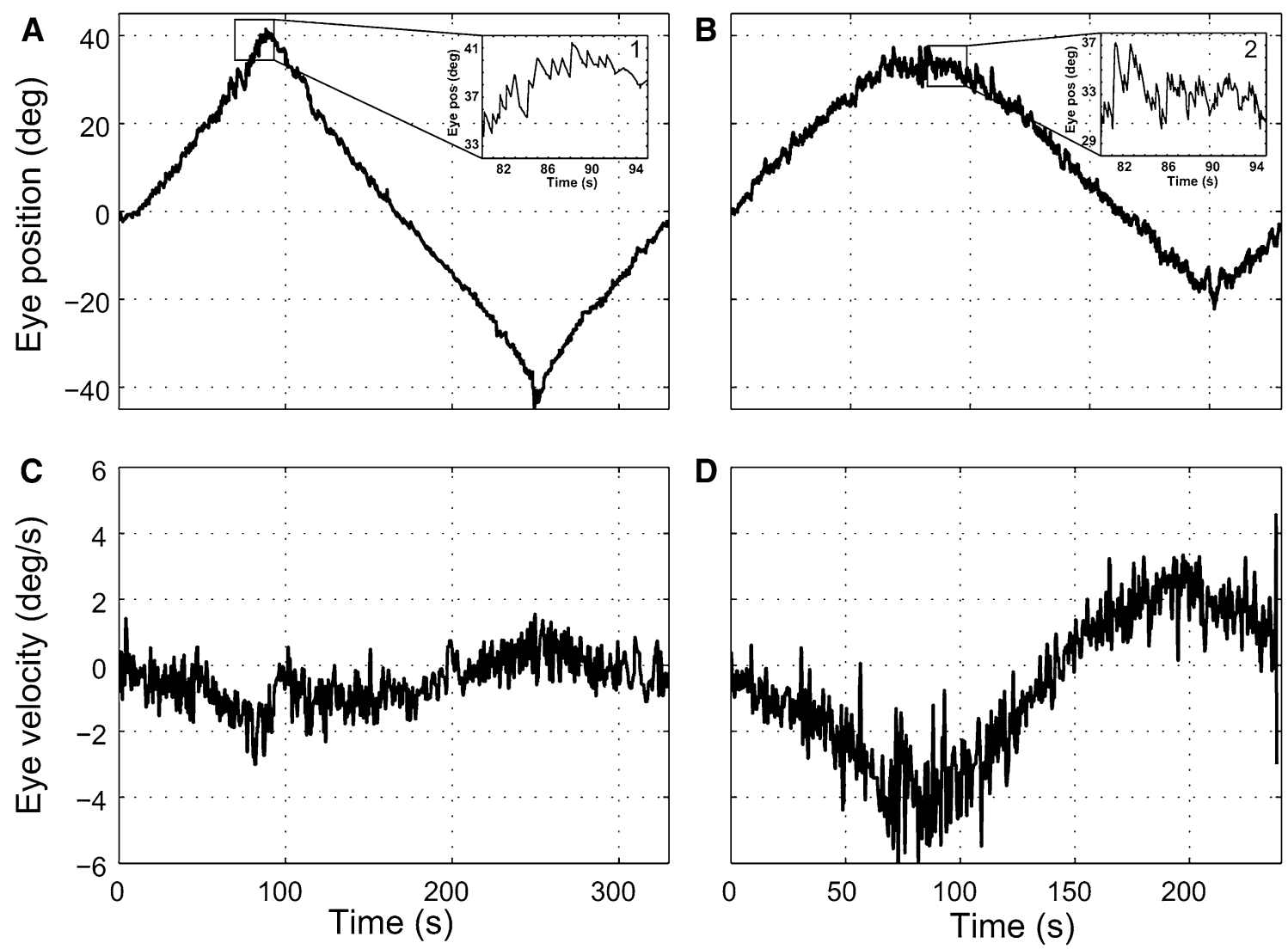

Fig. 3 Raw data recorded in a single trial from a control subject (a, c) and a typical patient $(\# 17 ; \mathbf{b}, \mathbf{d})$. Note that in the patient a single trial was shorter due to the smaller range of gaze covered $\left(60^{\circ} \mathrm{vs} .80^{\circ}\right.$, patients vs. controls) as one eye was covered. In $\mathbf{a}$ and $\mathbf{b}$ eye position is plotted as function of time. Positive angles correspond to right gaze eccentricities as seen by the subject. Inset 1 at extreme eccentricities the centrifugal beating nystagmus is clearly visible in the control

confirm such a pattern $\left[R^{2}=0.00, \quad p=0.98\right.$, slope $=0.0005(95 \%$ confidence interval -0.386 to $0.386)]$.

\section{Slow-moving dot paradigm vs. classic saccade-based paradigm}

The 12 bins corresponding to gaze eccentricities of $\leq 30^{\circ}$ were used to compare drift velocities obtained with the slow-moving dot and the saccade-based paradigm (see Fig. 5b, c for two representative patients). Eye-drift velocity recorded with the saccade-based paradigm was significantly higher for bins centered at $15^{\circ}(p<0.001$, nonparametric Wilcoxon signed-rank test) or larger. Pooling all bins and subjects, the mean ratio was $0.48 \pm 0.19$ (Table 2). We fitted the traces of the 13 patients who underwent both paradigms with Eq. (2) (results see legend of Fig. 5). By calculating the variation of the parameter values within each subject, we found it significantly different from 0 for $k_{2}$ [+62\% (9\%), p<0.001], but not for $k_{1}$

subject as well. Inset 2 at the same eccentricity of gaze as in inset 1, GEN in this patient is much stronger than in the controls. In $\mathbf{c}, \mathbf{d}$ eye velocity is plotted against time. The eye velocity begins to decrease from its baseline before the onset of the nystagmus, showing the growing centrifugal drift. Compared to the control subject (c), eye drift velocity in the patient (d) is considerably larger, indicating more GEN

[-23\% (44\%)], indicating that changes in the drift pattern between the two paradigms were driven by changes in scale only. Of the 18 patients characterized by an abnormal value of at least one parameter using the slow-moving dot paradigm, 12 were tested with the saccade-based paradigm and five showed a change in the classification: Three patients previously categorized as pure shape-changing (\#6, 15 and 20) were now classified as scaling and shapechanging. One patient (\#5) switched from pure scaling to combined scaling and shape-changing in the saccade-based paradigm, one patient (\#13) switched from combined scaling and shape-changing to pure scaling in the saccadebased paradigm.

\section{Discussion}

Gaze holding is provided by the inherently 'leaky' brainstem neuronal velocity-to-position integrator, whose performance is optimized by the vestibulo-cerebellum [5, 22]. 

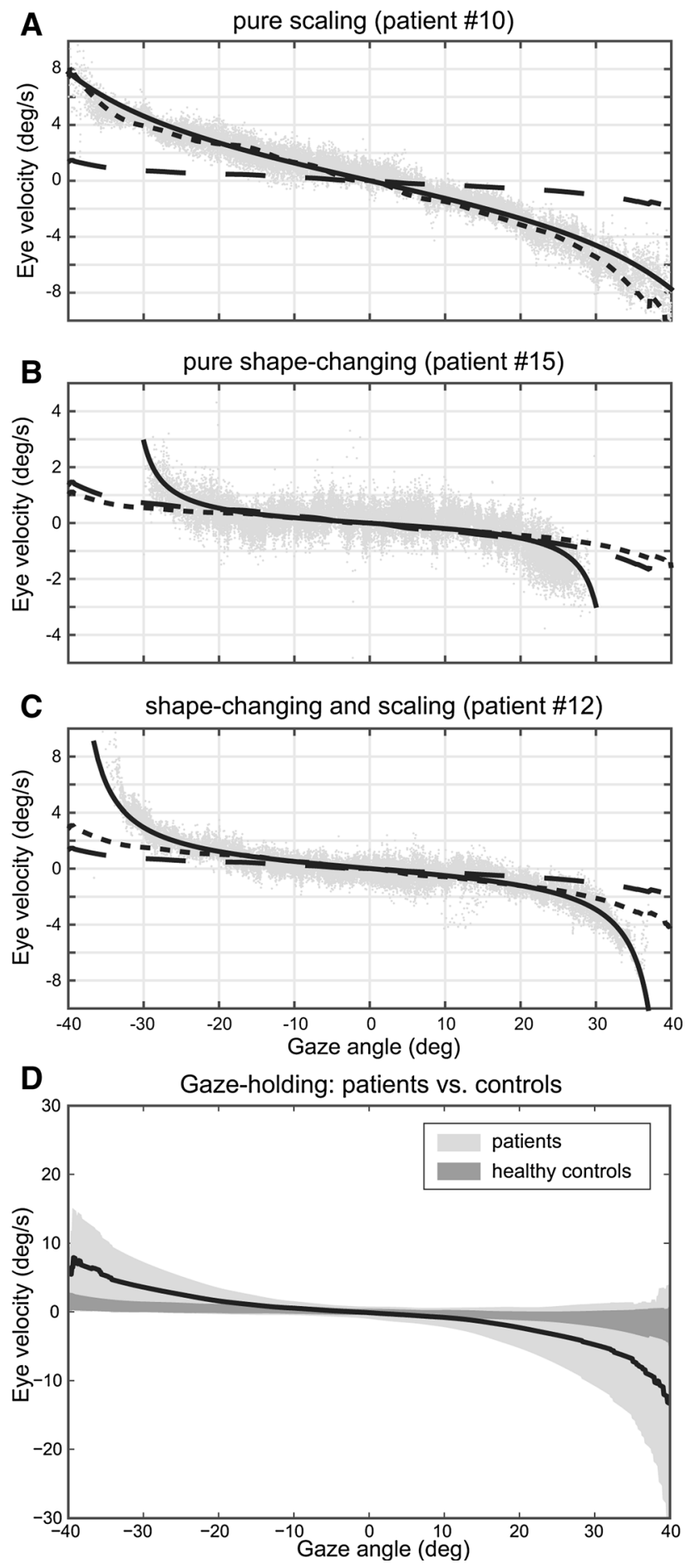

Gaze-evoked nystagmus (GEN) is considered an essential clinical sign of a deficient brainstem neural velocity-toposition integrator or its cerebellar modulatory structures. Here we discuss the results of 21 patients with neurodegenerative cerebellar disease that presented with impaired gaze holding to the Department of Neurology of the University Hospital Zurich, Switzerland. This study was
Fig. 4 PV-plots providing eye drift velocity for all gaze eccentricities tested illustrate the different drift behaviors in individual patients (ac) and pooled for all patients and controls (d). Both single raw data points (in gray) and the fit of the tangent function (black solid line) are shown for each patient. To set the drift behavior of the patients in relation to that of the healthy controls, the mean fit in the healthy controls is provided both as originally obtained (black dashed line) and scaled-up to match the eye drift velocity of the patient (black dotted line). For patients that showed an increase of eye drift velocity for all angles of gaze eccentricity, the scaled-up fit from the controls will match the patient's fit well (a). Likewise, in patients that presented with increased eye drift velocity only at larger angles of gaze eccentricity, the mean fit from the controls will match the patient's drift behavior within a certain range of drift angles without scaling, but for larger gaze angles, changes in shape are occurring (b). A combination of scaling and shaping was observed in some subjects as well (c). In d, the mean of the patients' position-velocity (PV) plots (black solid trace) $\pm 1 \mathrm{SD}$ (light-gray area) is presented for each gaze eccentricity tested. The dark gray shaded area refers to the healthy controls' mean trace \pm 1 SD. The eye drift velocity behaved similarly in the patient group and the control group, growing with gaze eccentricity and showing a pronounced non-linearity at large gaze eccentricities, with eye drift velocity in the patients being clearly higher. Average $( \pm 1 \mathrm{SD})$ peak eye drift velocity was $5.9^{\circ} / \mathrm{s}( \pm .5 \% \mathrm{~s})$ for the patients (obtained at $32.2 \pm 5.3^{\circ}$ of eccentricity)

driven by the hypothesis that patients with cerebellar disease will show profound non-linear eye drift at eccentric gaze and that a more thorough observation of the pattern of eye drift behavior may allow a distinction between different patient populations-probably linked to the underlying disease. Compared to controls, eye-drift velocity was significantly higher for gaze eccentricities as small as $\pm 20^{\circ}$, being consistent with previous reports describing GEN at eccentricities of $\pm 15^{\circ}$ in patients with cerebellar dysfunction [23]. We found the increase of centripetal eye-drift velocity as a function of gaze eccentricity to be markedly non-linear, reproducing the eye-drift behavior we observed at extreme lateral gaze-angles in healthy human subjects [19]. Fitting confirmed that it could best be approached by a tangent function.

We propose for the first time a link between the pattern of eye-drift behavior and age at disease-onset in patients with neurodegenerative cerebellar disease. Specifically, patients presenting with pure shape-changing of eye-drift behavior had significantly ( $p=0.001)$ earlier disease-onset compared to patients with pure scaling of eye-drift behavior. This global increase in eye-drift velocity in elderly patients with cerebellar degeneration cannot be explained by physiological age of cerebellar structures, as in our healthy control subjects we did not observe any increase in eye-drift velocity with age.

We believe that our detailed characterization of gaze holding is also of relevance for the clinical assessment of patients with suspected gaze-holding deficits, since it implies that testing a single gaze angle (as usually performed at the bedside) might be insufficient. Specifically, the current clinical approach bears the risk of under-estimating 
Table 3 Eye drift velocity-comparison between patients and controls and between the slow-moving dot paradigm and the saccade-based paradigm

\begin{tabular}{|c|c|c|c|c|c|c|c|c|c|}
\hline Gaze angle $\left(^{\circ}\right)(\text { center of bin })^{\mathrm{a}}$ & $40^{\mathrm{b}}$ & $35^{\mathrm{b}}$ & $30^{\mathrm{b}}$ & $25^{\mathrm{b}}$ & $20^{\mathrm{b}}$ & 15 & 10 & 5 & 0 \\
\hline \multicolumn{10}{|c|}{ Patients $(n=21)$ vs. controls $(n=20)$ : comparison of eye drift velocity (slow-moving dot paradigm only) $(\%$ s) } \\
\hline Patients & $4.3(2.8)$ & $3.7(2.4)$ & $2.7(1.6)$ & $2.2(1.3)$ & $1.4(0.8)$ & $0.8(0.6)$ & $0.4(0.5)$ & $0.1(0.3)$ & $0.1(0.3)$ \\
\hline Healthy subjects & $1.1(0.7)$ & $0.9(0.6)$ & $0.7(0.4)$ & $0.6(0.3)$ & $0.5(0.3)$ & $0.4(0.3)$ & $0.3(0.3)$ & $0.1(0.2)$ & $0.0(0.1)$ \\
\hline \multicolumn{2}{|l|}{ Gaze angle $\left(^{\circ}\right)(\text { center of bin })^{\mathrm{a}}$} & $30^{\mathrm{c}}$ & $25^{\mathrm{c}}$ & \multicolumn{2}{|c|}{$20^{\mathrm{c}}$} & \multicolumn{2}{|r|}{10} & 5 & 0 \\
\hline \multicolumn{10}{|c|}{ Patients only: comparison of eye drift velocity between the two paradigms $(n=13)(\% / \mathrm{s})$} \\
\hline \multicolumn{2}{|c|}{ Slow moving } & $2.6(0.9)$ & \multicolumn{2}{|c|}{$2.1(1.1) \quad 1$} & \multicolumn{2}{|c|}{$0.5(0.5)$} & $0.4(0.4)$ & $0.1(0.2)$ & $0.1(0.3)$ \\
\hline \multicolumn{2}{|l|}{ Saccade-based } & $3.7(1.6)$ & \multicolumn{2}{|c|}{$3.2(1.6)$} & \multicolumn{2}{|c|}{$1.4(1.0)$} & $0.7(0.7)$ & $0.3(0.4)$ & N/A \\
\hline
\end{tabular}

${ }^{a}$ Values from corresponding bins on right and left gaze were pooled

b At these angles median eye drift velocity was significantly $(p<0.001)$ larger in the patient group compared to the control group

${ }^{c}$ At these angles median eye drift velocity was significantly $(p<0.001)$ larger for the saccade-based paradigm compared to the slow-moving dot paradigm

or over-estimating impairments in gaze holding, depending on the gaze angle chosen and the drift behavior (scaling, shape-changing). We, therefore, propose more thorough testing including different gaze angles to determine the characteristics of GEN at the bedside.

\section{Different patterns of drift behavior in cerebellar degeneration}

Here we describe a non-linear relationship between eyedrift velocity and gaze eccentricity in patients with cerebellar neurodegeneration. As a result of this non-linearity a distinction between different gaze-dependent eye-drift behaviors can be made. From the 18 patients with significant GEN, scaling of the healthy subjects behavior was observed in 14 and changes in the shape of the gaze behavior in eight. While in four of these eight patients, changes in shape were accompanied by changes in scale, eye drift pattern in the remaining four only changed in shape, i.e. abnormal eye drift velocity was limited to the most eccentric gaze positions. Considering the two observed behaviors, it can be hypothesized that pure shape-changes reflect earlier stages of cerebellar degeneration, with gaze holding remaining sufficient over a broad range of (small and medium-sized) gaze angles, while later gaze holding becomes impaired globally as reflected by changes in scale. However, 'mild' disease-severity rating (Table 1) and shorter disease duration were found more frequently in the pure scaling group $(70 \%)$ compared to the pure shapechanging group $(50 \%)$, which argues against scaling reflecting more advanced/severe cerebellar dysfunction. Furthermore, disease duration was significantly ( $p=0.046)$ shorter in the pure scale-changing group compared to the pure shape-changing group. We, therefore, consider it unlikely that the drift pattern is related to the stage of disease. Noteworthy, mean age both at symptom- onset and at recording time in the pure shape-changing group was significantly $(p<0.05)$ lower than that in the pure scaling group. This raises the question whether the drift pattern (defining whether gaze-holding failure is global or restricted to far-lateral gaze) could be linked rather to the patient's age than to the disease duration. While we do see support for this hypothesis, we would like to emphasize that we found the same pure shape-changing GEN behavior in two affected brothers aged 35 and 31 years at symptom-onset (\#6 and \#15) with presumably autosomal-dominant cerebellar ataxia. This and the fact that the average age of symptom-onset varies considerably for different neurodegenerative cerebellar disorders lead us hypothesize that the various drift behaviors could be a consequence of distinct genetically defined cerebellar syndromes becoming symptomatic at different ages. This hypothesis, however, is limited by the fact that genetic testing was lacking in most of our patients and that the diagnosis remained descriptive.

\section{Only scale of GEN is affected by the specific paradigm applied}

While traditionally paradigms using saccades to reach static eccentric targets were applied to quantify GEN, the target used here was slowly displacing and flashing. Whereas this resulted in much higher spatial resolution and more robust measurements of the position-velocity (PV)plot, it also made GEN more prone to adaptation, since subjects' gaze remained at (large) angles of eccentricity over a prolonged period of time. Thereby the amount of GEN is likely underestimated by our paradigm. This explains also why the three pure shape-changing patients from the slow-moving dot paradigm were found to have additional (minor) scaling in the saccade-based paradigm. A subject-by-subject comparison between our paradigm 
A Segregation of the distinct drift behaviors

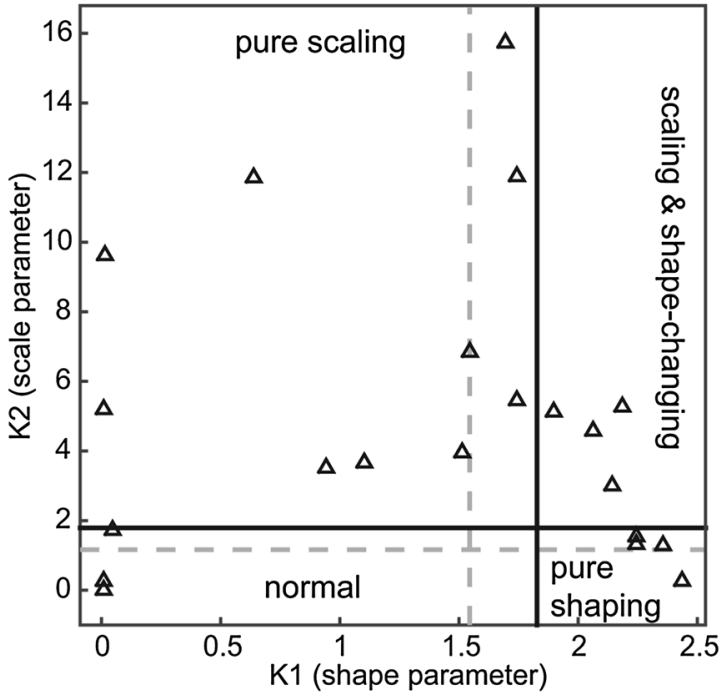

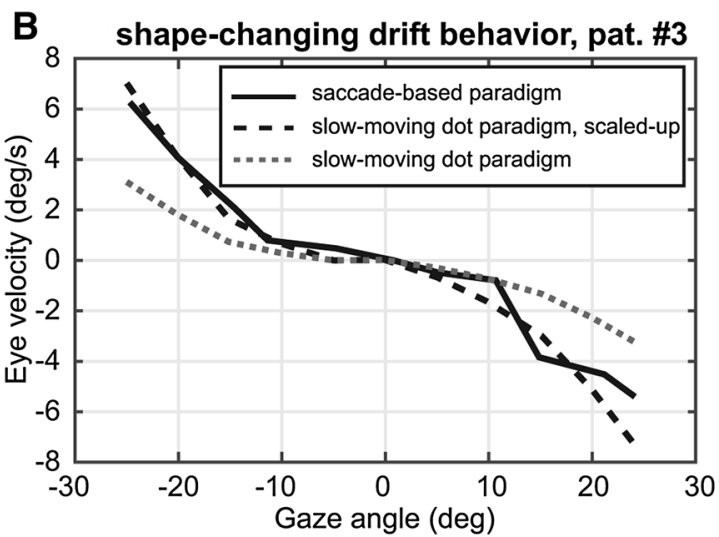

Fig. 5 a Segregation of the drift behavior based on the distribution of model parameters $k_{1}$ and $k_{2}$ in the 21 patients (referred to by triangles). Thereby each patient's drift pattern is classified as either pure scaling, pure shape-changing, combined scaling and shapechanging or within normal limits. Cutoff values (indicated by the solid black lines) for significantly increased $k_{1}$ and $k_{2}$ values are defined as values above the mean values (gray dashed lines) plus 1SD of the healthy controls. The median values for the parameters using the tangent fit were $k_{1}=1.69 \quad(0.55), k_{2}=3.94 \quad(2.39)$ and $c_{2}=-0.16(0.46)$ for patients and $k_{1}=1.54(0.28), k_{2}=1.17$ (0.62), $c_{2}=-0.02(0.29)$ for healthy subjects. A significant difference was observed for $k_{2}$ ( $p=0.002$, nonparametric Mann-WhitneyWilcoxon test), but not for $k_{1}$ and $c_{2}$. b, c Comparison of the PV-plot obtained with the slow-moving dot paradigm (gray dotted line) and obtained with the saccade-based paradigm (black solid line) in a

and the classic approach revealed that eye-drift velocity at bins centered at $\pm 15^{\circ}$ or larger was significantly $(p<0.001)$ lower, reaching in median $48 \%$ of the values obtained with the classical saccade-based paradigm. However, our fit revealed that these differences were related to changes in scale only. The resulting PV-plots, indeed, resemble in shape those obtainable from the classic saccade-based paradigm. Therefore, we can conclude that

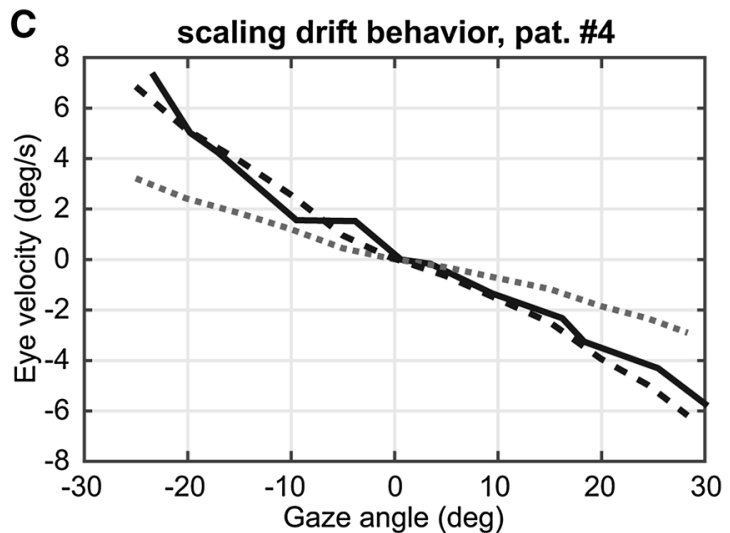

typical subject with a shaping-only drift behavior (b) and a subject with a pure scaling drift behavior (c). Since overall drift was stronger for the classic saccade-based paradigm, eye drift velocity for the slow-moving dot paradigm was scaled up accordingly to allow better comparison (black dashed line). The gray dotted line indicates the non-scaled eye drift velocity from the slow-moving dot paradigm. Fitting the traces of all 13 patients who underwent both paradigms with Eq. (2) resulted in the following median parameters for the saccade-based paradigm: $k_{1}=2.10(0.71), k_{2}=6.07$ (2.70) and $c_{2}=0.05(0.43)$. The $r^{2}$ was $0.96(0.03)$. The median of the parameters estimated on data acquired in the same range of gaze eccentricities from the slow-moving dot paradigm in same 13 subjects were $k_{1}=1.99(0.47), k_{2}=3.33(1.75)$ and $c_{2}=-0.01(0.32)$ $\left[r^{2}=0.95(0.04)\right]$

the pattern of eye-drift velocity is not paradigm-specific but reflects a general behavior of cerebellar dysfunction. Hence, as the main feature assigning patients to one of our groups was the presence/absence of a change in shape with respect to the normal PV-plot, the distinction between the two different observed behaviors holds. It must be taken into account, however, that using one or the other paradigm results in observing two different values of drift velocity. 
Accordingly, using the classical saccade-based paradigm, one should expect to observe patients with a pure scaling effect, forming one group, and patients showing a change in shape paired with a minor scaling effect, forming the second group. Therefore, in the saccade-based paradigm, the distinction between the three different groups identified in the slow-moving dot paradigm may be less clear. While the addition of minor scaling to pure shape-changing in three patients can be explained by less GEN adaptation in the saccade-based paradigm, the addition (\#5) or loss (\#13) of shape-changing in addition to scaling observed in two patients when using the saccade-based paradigm needs to be addressed further. In these two patients, such shifts in the shape-changing parameter are most likely paradigmrelated and could potentially be explained by higher uncertainty levels and smaller sample size (only few noncontinuous gaze angles measured) in the saccade-based paradigm, affecting the mathematical modeling used to determine scaling and shape-changing parameters.

From the clinical perspective, we advocate the use of the slow-moving dot paradigm in the evaluation of patients with vestibulo-cerebellar disease instead of the saccadebased paradigm, as with this quantitative approach a much more detailed characterization of gaze-holding deficits can be achieved. While overall eye-drift velocity values were lower in the slow-moving dot paradigm, this did not result in a lower rate of detection of gaze-holding impairments in the patients. Higher resolution of the slow-moving dot paradigm, therefore, will likely improve the identification of more subtle gaze-holding deficits; however, it needs further confirmation in future studies to become the new "gold standard" in the evaluation of gaze-holding deficits in cerebellar loss of function.

\section{Distribution of cerebellar atrophy in patients with GEN}

Based on the MRI-assessment, we propose a characteristic pattern of distribution of cerebellar atrophy in patients with impaired gaze holding $(n=18 / 21)$ due to neurodegenerative cerebellar disease. Almost invariably $(n=15 / 18)$ we noted involvement of the superior and dorsal vermis (especially lobules IV-VIIb). Additional areas of atrophy in these 15 patients with vermal atrophy included the anterior lobe of the cerebellar hemispheres $(n=10 / 15)$, the flocculus/paraflocculus $(n=7 / 15)$ and the dentate nucleus ( $n=9 / 15$ ). The caudal parts of the vermis (uvula, nodulus) were usually spared. We did not identify distinct patterns of cerebellar atrophy related to the eye-drift behavior observed.

In the literature there is conflicting information about the cerebellar gaze-holding network. With floccular/parafloccular involvement in half of the patients, our data are in agreement with results from lesion studies in non-human primates identifying the flocculus as key structure for gaze holding [13]. In accordance with our observation, the dentate nucleus has previously been identified as part of the cerebellar gaze-holding network [24]. Whether the superior and dorsal vermal lobules contribute to gaze holding is not clear. The lobules of the superior vermis (lobules I to V) are known to integrate vestibular and proprioceptive input [25] and are likely important for postural stability [26], while lobules VI and VII contribute to smooth pursuit and saccades [27]. Noteworthy, partial midline/paramedian cerebellectomy including vermal lobules IV-IX, but sparing the dentate nucleus and the flocculus/paraflocculus, did not result in GEN in two cats [16]. These studies, therefore, suggest that the superior and dorsal vermal lobules do not belong to the cerebellar gaze-holding network and rather propose that this network relies on other cerebellar structures, such as the dentate nucleus [24] or the flocculus/paraflocculus [13].

Noteworthy, the quality and resolution of MRI varied among individual participants, which may have limited the MRI-ratings. Furthermore, the amount of cerebellar atrophy in patients presenting with cerebellar dysfunction strongly depends on the underlying disease. In two brothers with suspected ADCA both MRI findings and GEN patterns were very similar, suggesting that a given underlying genotype may indeed be reflected in a characteristic phenotype of cerebellar gaze-holding impairment.

In conclusion, we confirm that in patients with neurodegenerative disease the increase of GEN as a function of gaze eccentricity is markedly non-linear. Gaze-angles as small as $20^{\circ}$ resulted in significantly increased amplitudes of GEN compared to healthy human subjects, reflecting the insufficiently compensated leakiness of the neuronal velocity-to-position integrator. We emphasize that the observed age-dependent differences in the drift behavior (pure shape-changing in the young vs. pure scaling in the elderly) are likely disease-specific with underlying disorders becoming symptomatic at distinct ages. This potentially allows the discrimination between early- and lateonset cerebellar neurodegeneration based on eye-drift patterns, but requires examination of gaze holding at different angles of gaze eccentricity. Whether this will allow the distinction of different cerebellar neurodegenerative disorders based on gaze-holding deficits or not will be subject to future studies in patients with genetically confirmed, cerebellar disorders.

Acknowledgments Dr. Tarnutzer and Dr. Bertolini were supported by the Swiss National Science Foundation, the Betty and David Koetser Foundation for Brain Research and the Zurich Center for Integrative Human Physiology, Switzerland. The authors thank Marco Penner for technical support. 
Conflict of interest The authors declare that they have no competing interests.

\section{References}

1. Stahl JS, Averbuch-Heller L, Leigh RJ (2000) Acquired nystagmus. Arch Ophthalmol 118:544-549

2. Holmes G (1907) A form of familial degeneration of the cerebellum. Brain 30:466-488

3. Cannon SC, Robinson DA (1987) Loss of the neural integrator of the oculomotor system from brain stem lesions in monkey. J Neurophysiol 57:1383-1409

4. Zee DS, Leigh RJ, Mathieu-Millaire F (1980) Cerebellar control of ocular gaze stability. Ann Neurol 7:37-40

5. Robinson DA (1974) The effect of cerebellectomy on the cat's vestibulo-ocular integrator. Brain Res 71:195-207

6. Godaux E, Cheron G (1996) The hypothesis of the uniqueness of the oculomotor neural integrator: direct experimental evidence in the cat. J Physiol 492(Pt 2):517-527

7. Lee H, Kim HA (2012) Nystagmus in SCA territory cerebellar infarction: pattern and a possible mechanism. J Neurol Neurosurg Psychiatry

8. Tarnutzer AA, Berkowitz AL, Robinson KA, Hsieh YH, Newman-Toker DE (2011) Does my dizzy patient have a stroke? A systematic review of bedside diagnosis in acute vestibular syndrome. CMAJ 183:E571-E592

9. Zee DS, Yee RD, Cogan DG, Robinson DA, Engel WK (1976) Ocular motor abnormalities in hereditary cerebellar ataxia. Brain 99:207-234

10. Baloh RW, Konrad HR, Honrubia V (1975) Vestibulo-ocular function in patients with cerebellar atrophy. Neurology 25:160-168

11. Hood JD, Kayan A, Leech J (1973) Rebound nystagmus. Brain 96:507-526

12. Leech J, Gresty M, Hess K, Rudge P (1977) Gaze failure, drifting eye movements, and centripetal nystagmus in cerebellar disease. Br J Ophthalmol 61:774-781

13. Zee DS, Yamazaki A, Butler PH, Gucer G (1981) Effects of ablation of flocculus and paraflocculus of eye movements in primate. J Neurophysiol 46:878-899

14. Burde RM, Stroud MH, Roper-Hall G, Wirth FP, O'Leary JL (1975) Ocular motor dysfunction in total and hemicerebellectomized monkeys. Br J Ophthalmol 59:560-565
15. Westheimer G, Blair SM (1973) Oculomotor defects in cerebellectomized monkeys. Invest Ophthalmol 12:618-621

16. Optican LM, Robinson DA (1980) Cerebellar-dependent adaptive control of primate saccadic system. J Neurophysiol 44:1058-1076

17. Eizenman M, Cheng P, Sharpe JA, Frecker RC (1990) End-point nystagmus and ocular drift: an experimental and theoretical study. Vision Res 30:863-877

18. Shallo-Hoffmann J, Schwarze H, Simonsz HJ, Muhlendyck H (1990) A reexamination of end-point and rebound nystagmus in normals. Invest Ophthalmol Vis Sci 31:388-392

19. Bertolini G, Tarnutzer AA, Olasagasti I, Khojasteh E, Weber KP, Bockisch CJ, Straumann D, Marti S (2013) Gaze holding in healthy subjects. PLoS One 8:e61389

20. Dera T, Boening G, Bardins S, Schneider E (2006) Low-latency video tracking of horizontal, vertical, and torsional eye movements as a basis for 3-DOF realtime motion control of a headmounted camera. IEEE Conf Syst Man Cybern 6:5191-5196

21. Schneider E, Dera T, Bard K, Bardins S, Boening G, Brandt T (2005) Eye movement driven head-mounted camera: it looks where the eyes look. IEEE Conf Syst Man Cybern 3:2437-2442

22. Leigh RJ, Zee DS (2006) The neurology of eye movements, 4th edn. Oxford University Press, New York

23. Versino M, Hurko O, Zee DS (1996) Disorders of binocular control of eye movements in patients with cerebellar dysfunction. Brain 119(Pt 6):1933-1950

24. Baier B, Dieterich M (2011) Incidence and anatomy of gazeevoked nystagmus in patients with cerebellar lesions. Neurology 76:361-365

25. Manzoni D, Andre P, Pompeiano O (2004) Proprioceptive neck influences modify the information about tilt direction coded by the cerebellar anterior vermis. Acta Otolaryngol 124:475-480

26. Goldberg JM, Wilson VJ, Cullen KE, Angelaki DE, Broussard DM, Büttner-Ennever JA, Fukushima K, Minor LB (2012) The vestibular system: a sixth sense. Oxford University Press, New York

27. Lewis RF, Zee DS (1993) Ocular motor disorders associated with cerebellar lesions: pathophysiology and topical localization. Rev Neurol (Paris) 149:665-677

28. Schmahmann JD, Doyon J, McDonald D, Holmes C, Lavoie K, Hurwitz AS, Kabani N, Toga A, Evans A, Petrides M (1999) Three-dimensional MRI atlas of the human cerebellum in proportional stereotaxic space. NeuroImage 10:233-260 